at Columbus, Ohio, ${ }^{2}$ was given by Goldberg, McMath, Mohler and Pierce ${ }^{1}$ who assumed a variation of the carbon monoxide content of the earth's atmosphere with geographical location. To investigate this problem further, tracings of both the fundamental and first overtone regions, $4.7 \mu$ and $2.3 \mu$, respectively, were taken within a short time interval with the newly installed solar spectrometer of the Dominion Observatory at Ottawa. The fundamental band was obtained with about the same intensity as at Columbus. The terrestrial lines in the overtone band were barely observable, their intensity being comparable to the upper limit estimated for the intensities at Lake Angelus and Mt. Wilson.

The observations at Ottawa lead therefore to the same atmospheric concentration of carbon monoxide as at Columbus and show that the same concentration could also be present at Lake Angelus and Mt. Wilson. Moreover we find that the observed high intensity ratio of the fundamental and first overtone bands is quite expected from the laboratory absorption coefficients given by Penner and Weber. ${ }^{3}$ We conclude, therefore, that there is at present no reason to assume a geographical variation of atmospheric carbon monoxide.

Using the laboratory values of the absorption coefficients we compute the abundance of carbon monoxide in the atmosphere to be $0.06 \mathrm{~cm}$. atmos. The calculated abundance of carbon monoxide in the atmosphere and the intensity ratio of the two bands were confirmed by laboratory tracings taken with the same instrument using an absorption cell filled with air at pressure 0.5 atmos. and an amount of carbon monoxide equivalent to that calculated for the atmosphere.

I. L. Goldberg, R. R. McMath, O. C. Mohler, and A. K. Pierce, Phys. Rev. 85, 48I, I952.

2. J. H. Shaw, R. M. Chapman, J. N. Howard, and M. L. Oxholm, Ap. J. I I3, 268, I95I.

3. S. S. Penner and D. Weber, J. Chem. Phys. I9, 807, I95I.

Dominion Observatory, Ottawa, Canada.

\section{McDonald, J. K. Hydrogen absorption lines in the spectra of B-type stars.}

This investigation is an attempt to use the characteristic difference in the shapes and intensities of the hydrogen lines in the spectra of B-type stars as a criterion of the distances of the stars, by predicting theoretically from modelatmosphere computations the profiles of the hydrogen lines as a function of surface gravity and temperature, and then relating these results to the observed profiles in the spectra of B-type stars of known distance.

A model stellar atmosphere was computed for an assumed effective temperature, $20,700^{\circ} \mathrm{K}$; assumed gravity, $\log g=3.80$; and assumed abundances of the elements by weight, hydrogen: helium: heavier elements $=$ I000:800:50. The condition of radiative equilibrium was found to be well satisfied for the model. The computed flux was constant within \pm 4 per cent, for optical depths from 0.00 to 2.00 , and this constancy of flux was found to be relatively insensitive to alterations in the assumed grey-body temperature distribution. The energy distribution in the continuous spectrum of the model indicated a Balmer discontinuity of 0.105 , a bolometric correction of -2.63 , and a colour temperature on the Greenwich scale of $35,000^{\circ} \mathrm{K}$. The value of Balmer discontinuity and the computed number of observable hydrogen lines, when compared with observations, supported the assumption that the model approximates a main-sequence B2 star. The profile of the $H \gamma$ line was computed for the model, assuming two different re-emission processes to be operating, monochromatic radiative equilibrium (pure scattering) and local thermodynamic equilibrium (pure absorption). The computed profiles were compared with the observed profiles of $H_{\gamma}$ in the spectra of four stars in the range $\mathrm{B}_{\mathrm{I}}-\mathrm{B}_{3}$, III or IV. It appears that the contour of the wings of the lines, computed by either of the assumed processes, represents rather well the observed profile for a star of type B2.5 IV, but neither process yields a good representation of the line centre.

The conclusion is that the currently used model-atmosphere method of computing the $\mathrm{H \gamma}$ line profile will not provide a sufficiently discriminating criterion of the absolute magnitudes of the B-type stars, but an extension of the investigation to additional models may possibly modify this conclusion.

\section{Dominion Astrophysical Observatory,
Victoria, B. C.}

\section{McLaughlin, Dean B. The variable profiles of emission bands of Nova Aquilae IgI8.}

During secondary oscillations of light, novae show higher excitation at minima than at maxima. This is attributed to variation of temperature of the effective photosphere of the central star. Several novae have had smooth emission bands at maxima of light, and a pattern of 
maxima and minima in the bands during light minima. This is assigned to variable penetration of excitation into the ejected shell, revealing the true distribution of matter best during light minima. ${ }^{1}$

In Nova Aquilae the bands showed strong central maxima during light maxima, and prominent "horns" at the band edges in the light minima. ${ }^{2}$ From the later pattern of the nebula, it is clear that the minima represent more nearly the true distribution of matter.

Baade's proposed system of rings and polar caps as a model of the Nova Aquilae nebula gives an excellent account of the Doppler patterns observed with the slit in different position angles. ${ }^{3}$ The central maxima can be assigned to an "equatorial ring," closer to the star than most of the gas; the "horns" are assigned to "polar caps" ejected towards and from the earth at an angle of $16^{\circ}$ to the line of sight. The ring was a small fraction of the total ejection, while the polar caps were a large fraction.

It is suggested that, during light minima, the star's radiation was able to excite all the ejected gas, even the dense polar knots, which were then brought out in their correct proportions. At the light maxima, the star's cooler radiation could excite the entire equatorial ring and the inner sides of the polar knots, but could not penetrate far into the latter; hence the central maxima were accentuated and the horns suppressed. The forbidden emissions, especially $[N \mathrm{II}]$, had the central maxima stronger relative to the horns than did hydrogen and other permitted lines. This is consistent with higher density and resulting greater strength of permitted lines in the polar knots.

I. McLaughlin, Pub. A. S. P. 6o, 54, 1948.

2. Payne and Menzel, Pub.A.A.S. 8, I8, 1934; Sayer, Circ. Harv. Coll. Obs. No. 406, I 935.

3. Unpublished, but see van de Hulst, Problems of Cosmical Aerodynamics, p. II6, Central Air Documents Office, I95I.

University of Michigan Observatory, Ann Arbor. Mich.

\section{Miller, Freeman D. Rank correlation in astro- nomical statistics.}

Conventional correlation methods are not applicable to statistical problems in which a physical or geometrical variable is represented by a ranking in order of its intensity of development, rather than by a series of measurements. Such characteristics are not uncommon in astronomy: the spiral classification $\mathrm{Sa}, \mathrm{Sb}, \mathrm{Sc}$; the classifica- tion of solar flares into "importance" groups; and the spectral classifications of the Henry Draper Catalog are familiar examples of ranked data.

It is frequently necessary to correlate such variables with one another, or with measured data. Conventional correlation is sometimes (incorrectly) employed, or, more often, resort is had to simple inspection of graphs. Astronomically, no use appears to have been made of the method of rank correlation, a theory developed precisely for the purpose of statistical discussion of ranked data. ${ }^{1,2,3}$ In this theory, the correlation coefficient, its mean error, and the estimate of significance, which are familiar features of the conventional theory, have their analogs for ranked data.

Details of applications to several sets of astronomical data will appear elsewhere.

I. M. G. Kendall, Rank Correlation Methods, 1948.

2. H. Jeffreys, Theory of Probability, 1939.

3. Symposium on Ranking Methods; J. R. Statist. Soc., Series B, I 2, I 53, I950.

Univ. of Michigan Observatory, Ann Arbor, Michigan.

\section{Nail, Virginia McKibben. Eclipsing variable stars in the Magellanic Clouds.}

In the Small and Large Magellanic Clouds we have found and studied twenty-four and twentyone high luminosity eclipsing stars, respectively. Through analyses of eclipsing star population in fourteen neighboring comparison variable star fields, all but two or three of these stars are shown to be actual members of the Clouds. The periods range from 66.9 to I.O2 days, with the majority of periods coming in the interval between two and four days. The mean absolute magnitude is around -2 , and the $\beta$ Lyrae type predominates. Secondary minima are recorded for three-fourths of the variables. There is no clear indication of a concentration of eclipsing stars to the densest parts of the Clouds. Details are to be published from the Harvard Observatory in the near future.

Harvard College Observatory, Cambridge, Mass.

Odgers; G. J. and R. Stewart. Dynamics of turbulent flow and the evolution of stellar systems.

On examination most of the theories of binary star origin show various unsatisfactory features. It is unlikely for instance that fission can give rise to a star at all, and the accretion hypothesis runs into the difficulty that there is no correlation between the mass and period of a binary system. 